OnLine Journal of Biological Sciences 11 (3): 96-100, 2011

ISSN 1608-4217

(C) 2011 L. Danisovic et al., This open access article is distributed under a Creative Commons Attribution

(CC-BY) 3.0 license

\title{
The Effect of Salivary Gland Extract of Lucillia Sericata Maggots on the Human Dermal Fibroblasts Proliferation
}

\author{
${ }^{1}$ Simona Polakovicova, ${ }^{1}$ Stefan Polak, \\ ${ }^{2}$ Marcela Kuniakova, ${ }^{3}$ Marek Cambal, ${ }^{4}$ Juraj Majtan, \\ ${ }^{4}$ Peter Takac, ${ }^{4}$ Milan Kozanek and ${ }^{2,5}$ Lubos Danisovic \\ ${ }^{1}$ Institute of Histology and Embryology, \\ ${ }^{2}$ Institute of Medical Biology, Genetics and Clinical Genetics, \\ Faculty of Medicine, Comenius University in Bratislava, \\ Sasinkova 4, 81108 Bratislava, Slovakia \\ ${ }^{3}$ Department of Surgery, \\ Faculty of Medicine, Comenius University in Bratislava, \\ Mickiewiczova 13, SK-813 69, Bratislava, Slovakia \\ ${ }^{4}$ Institute of Zoology, Slovak Academy of Sciences, \\ Dúbravská Cesta 9, 84506 Bratislava, Slovakia \\ ${ }^{5}$ Institute of Laboratory Medicine, \\ St. Elizabeth University of Health and Social Sciences, \\ Palackého 1, 81000 Bratislava, Slovakia
}

\begin{abstract}
Problem statement: The aim of study was to investigate the effect of Salivary Gland Extract (SGE) of Lucillia sericata maggots on Human Neonatal Fibroblasts (B-HNF-1) proliferation and migration within collagen/hyaluronan membrane. Chronic, non-healing wounds are major health care problem worldwide. Nowadays, Maggot Debridement Therapy (MDT) has attracted much attention due to its successfull application in a treatment of chronic wounds where conventional treatment has failed. Maggot Excretion/Secretion (ES) contains specific proteinases which are positivelly involved in the remodeling of Extracelallar Matrix (ECM) components. Approach: Immunohistochemical methods using primary monoclonal antibody against marker vimentin was employed for the visualization of fibroblasts cultured within collagen hyaluronan membrane. Results: We found that maggot SGE at concentration of 2.4 and $4.8 \mathrm{gL} \mathrm{mL}^{-1}$ significantly induced the migration of fibroblasts into the membrane after 5 and 10 days of cultivation. On the other hand, SGE at concentration of $7.2 \mathrm{gL} \mathrm{mL}^{-1}$ reduced the migration of fibroblasts. Conclusion: The obtained results suggest that medicinal maggots secretion may speed up the wound healing process through the stimulation of fibroblast migration. However, further detailed research is needed to fully elucidate the substance (s) responsible for fibrloblast stimulation and migration.
\end{abstract}

Key words: Maggot therapy, biomaterial, cell culture

\section{INTRODUCTION}

Sterile maggots of greenbottle blowfly Lucillia (phaenicia) sericata (L. sericata) are applied to chronic wounds where conventional treatment has failed. This re-emerging treatment known as a Maggot Debridement Therapy (MDT) is used worldwide due to its efficacy, safety and simplicity. Mechanisms of action of maggots on wound healing include three beneficial effects: debridement, disinfection and enhancement of wound healing (Falch et al., 2009).

Larvae feed on the dead tissue, cellular debris and serous drainage of corpses or necrotic wound. They secrete proteolytic enzymes including collagenase, trypsin-like and chymotripsin-like enzymes, which take part in the breakdown of macromolecules on the wound surface (Beasley and Hirst, 2004). Debridement is aided by ingestion of the tissue and also may be enhanced by sinkova 4, 81108 Bratislava, Slovakia Tel: 00421259357215 
crawling, probing and macerating it with maggot mouthhooks (Sherman et al., 2000).

The movement and irritation caused by larvae ingesting liquefied necrotic tissue promotes the formation of a serous exudate by the wound, which causes lavage and dilution of bacterial concentration. Moreover, maggots digest the bacteria associated with tissue debris. They raises wound $\mathrm{pH}$ level from acid to alkaline, which inhibits the growth of bacteria, in particular Staphylococcus aureus and Group A and B streptococci (Whitaker et al., 2007).

The machanical action of the larvae irritating tissue stimulates the formation of granulation tissue. Allantoin, which is a component of larval secretions, provides an optimal environment for the growth of cells needed for wound healing. Larvae also secrete proteinases including serineproteinase, metalloproteinase and aspartylproteinase (Chambers et al., 2003; Horobin et al., 2006). These proteinases play a role in remodeling of extracellular matrix (Beasley and Hirst, 2004). Proteinases derived from many sources, particularly from fibroblasts, modulate cell surface receptors via direct activation influencing fibroblast proliferation and angiogenesis (Horobin et al., 2006). Furthermore, they may contribute in a proteolytic breakdown of ECM components by indirect activation, most notably fibronectin and induce fibroblast migration, chemotaxis reepitelisation and tissue remodeling (Gupta, 2008).

Horobin et al. (2006) developed a novel threedimensional in vitro model to observe fibroblast migration and morphology in response to maggot Excretion/Secretion (ES). This model more closely represented the microenvironment in which cells are present in vivo, provides for a much better understanding of the importance of interactions between the ECM, resident cells and ES in the wound healing process. They used populations of human foreskin fibroblasts embedded within gels composed of collagen and fibronectin. Autors investigated the effect of various concentrations of ES upon behavior and morphology of the fibroblasts. It has been shown that maggot ES stimulated and modified fibroblast migration in a dose-dependent manner in a comparison with relevant controls. Similarly, another studies (Plas et al., 2009a; 2009b) investigated the potential of maggots to modulate the secretion of cytokines under in vitro conditions. Plas et al. (2009a) showed that freshly isolated monocytes incubated with ES elevated the production of cytokine such as IL-8, IL-10 and growth factor beta-FGF but downregulated the production of TNF- $\alpha$ and IL-12p40.

The present study was focused on monitoring of effect of homogenate substances from salivary glands of $L$. sericata on the human dermal fibroblasts proliferation within collagen/hyaluronan membranes.

\section{MATERIALS AND METHODS}

Preparation of maggot salivary gland extract: In a total of 240 intact pairs of salivary glands were dissected from maggots of $L$. sericata and subsequently

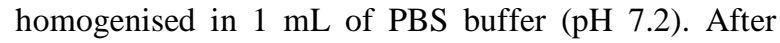
centrifugation of homogenate at $14000 \mathrm{rpm}$ for $30 \mathrm{~min}$, the supernatant was aliquoted and stored at $-20^{\circ} \mathrm{C}$. Before experiments, SGE at concentrations of 2.4, 4.8 and $7.2 \mathrm{gL} \mathrm{mL}^{-1}$ was prepared by diluting in Eagle's Minimal Essential Medium (E-MEM) (PAA, Austria) supplemented with $10 \%$ Fetal Calf Serum (FCS) (PAA, Austria) and gentamicin (Lék, Slovenia) in final concentration of $80 \mu \mathrm{g} \mathrm{mL} \mathrm{m}^{-1}$.

Cell culture: Cell culture of human neonatal fibroblasts B-HNF-1 (Repiska et al., 2010) was used in this study. Cells were thawed and cultured in complete E-MEM culture medium and cultured at $37^{\circ} \mathrm{C}$ in a humidified atmosphere containing $5 \% \mathrm{CO}_{2}$. Fibroblasts from third passage after thawing were seeded on the Collagen Hyaluronan Membrane (CHM).

Collagen Hyaluronan Membrane (CHM): The preparation of $\mathrm{CHM}$ was published previously (Vojtassak et al., 2001). The basic components of CHM were atelocollagen type I (Hypro, Czech Republic) and bacterial Hyaluronic Acid (HA) produced by Streptococcus zooepidermicus (Contipro, Czech Republic). Insoluble fibrous atelocollagen was mechanically treated in $0.5 \mathrm{M}$ acetic acid $(\mathrm{pH} 3.2)$. Water solution of HA was added to the acidic atelocollagen dispersion. The mixture was allowed for $15 \mathrm{~min}$ to form the complex. Obtained gel was dried on a teflon support at $37^{\circ} \mathrm{C}$. The HA content in the complex with atelocollagen was $8 \%(\mathrm{w} / \mathrm{w})$. The protein content was less than $0.1 \%$. Scaffolds used in all experiments were sized to $1 \mathrm{~cm}^{2}$ with the thickness of $0.3 \mathrm{~mm}$ and were sterilized with a dose of $28 \mathrm{kGy}$ using a ${ }^{60} \mathrm{Co}$ irradiation source.

Fibroblasts proliferation on the CHM: Sterile collagen/hyaluronan membranes were placed into 24well plate. They were covered with complete culture medium and stored in $\mathrm{CO}_{2}$ incubator overnight. After that, cell culture of B-HNF-1 was seeded on the surface of each CHM $\left(1 \times 10^{5}\right.$ cells/CHM). Extract in a concentration of $2.4,4.8$ and $7.2 \mathrm{gL} \mathrm{mL}^{-1}$ respectively was added. Fibroblasts without SGE were used as a negative control. Viability of fibroblasts on the membranes were continuously controled under inverted 
microscope Zeiss Axiovert 100 (Carl Zeiss, Germany) by using solution of 4',6-Diamidino-2-Phenylindole (DAPI) (Sigma-Aldrich, Germany). Fibroblasts with membranes were cultured 10 days and medium supplemeted with SGE was refreshed in $48 \mathrm{~h}$ interval. After 5 and 10 days of cultivation, cell culture medium was aspirated and membranes were rinsed with PBS and fixed.

Preparation of samples for immmunohistochemical examination: Samples selected for immunohistochemical examination were fixed in neutral formalin (Sigma-Aldrich, Germany) and embedded into paraffin. After that they were sectioned into $2 \mu \mathrm{m}$ thick slices and stained by conventional staining methods. Immunohistochemical staining was performed using the biotin-avidin technique with primary mouse antibodies against vimentin (DAKO, Glostrup, Denmark). Visualization of the cells was developed with DAKO LSAB/HRP kit and DAKO En Vision/HRP kit (DAKO, Glostrup, Denmark). Analysis was performed on the light microscope Nikon eclipse $80 \mathrm{i}$ and camera Nikon DS-Fi1.

\section{RESULTS}

Immunohistochemical examination after 5 and 10 days of the fibroblasts cultivation with SGE at selected concentrations revealed abundant fibroblasts on the surface of CHM formed one confluent layer or somewhere more than one layer (Fig. 1a, 2a and 3a).

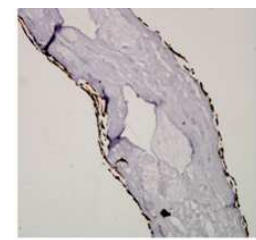

(a)

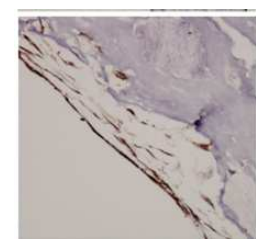

(c)

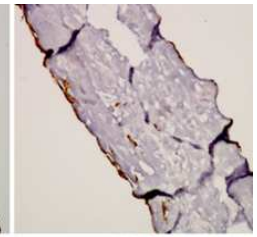

(b)

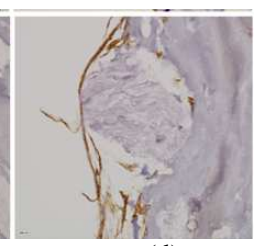

(d)
Fig. 1: Immunohistochemical examination of fibroblasts on the surface of collagen hyaluronan membrane after $5(\mathrm{a}, \mathrm{b})$ and 10 days $(\mathrm{c}, \mathrm{d})$ of the cultivation with $2.4 \mathrm{gL} \mathrm{mL}^{-1}$ of Salivary Gland Extract (SGE). Immunohistochemical staining was performed using the biotin-avidin technique with primary mouse antibodies against vimentin. (a) $\times 100$ (b) $\times 200$ (c) $\times 200$ (d) $\times 400$
The fibroblasts were orientated on the CHM longitudinaly. SGE at concentration of $2.4 \mathrm{gL} \mathrm{mL}^{-1}$ was able to induce the migration of limited numbers of fibroblasts into the CHM (Fig. 1). Immunohistochemical examination after fibroblasts cultivation with SGE at concentration of $4.8 \mathrm{gL} \mathrm{mL}^{-1}$ showed that the most of fibroblasts changed the orientation and migrated into the CHM (Fig. 2).
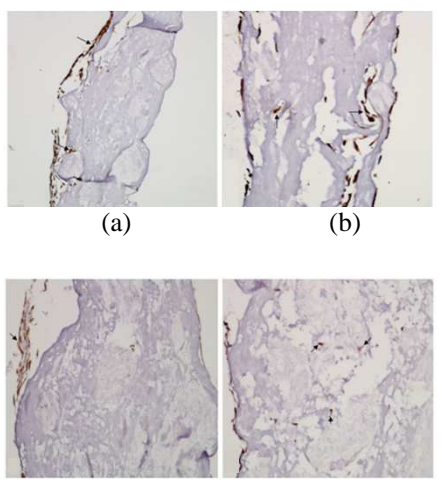

(c)

(d)

Fig. 2: Immunohistochemical examination of fibroblasts on the surface of collagen hyaluronan membrane after $5(\mathrm{a}, \mathrm{b})$ and 10 days $(\mathrm{c}, \mathrm{d})$ of the cultivation with $4.8 \mathrm{gL} \mathrm{mL}^{-1}$ of Salivary Gland Extract (SGE). Immunohistochemical staining was performed using the biotin-avidin technique with primary mouse antibodies against vimentin.

(a) $\times 100$ (b) $\times 200$ (c) $\times 200$ (d) $\times 200$

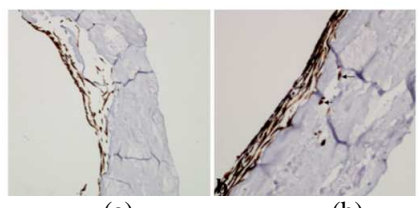

(a)

(b)

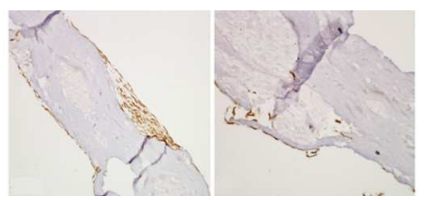

(c)

(d)

Fig. 3: Immunohistochemical examination of fibroblasts on the surface of collagen hyaluronan membrane after $5(\mathrm{a}, \mathrm{b})$ and 10 days $(\mathrm{c}, \mathrm{d})$ of the cultivation with $7.2 \mathrm{gL} \mathrm{mL}^{-1}$ of Salivary Gland Extract (SGE). Immunohistochemical staining was performed using the biotin-avidin technique with primary mouse antibodies against vimentin. (a) $\times 40$ (b) $\times 100$ (c) $\times 100$ (d) $\times 100$ 
OnLine J. Biol. Sci., 11 (3): 96-100, 2011

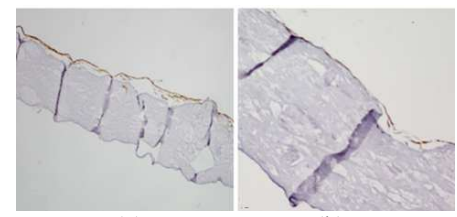

(a)

(b)

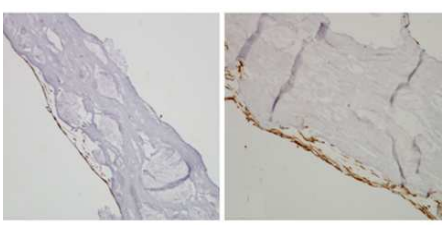

(c)

(d)

Fig. 4: Immunohistochemical examination of fibroblasts on the surface of collagen hyaluronan membrane after $5(\mathrm{a}, \mathrm{b})$ and 10 days $(\mathrm{c}, \mathrm{d})$ of the cultivation without Salivary Gland Extract (SGE) (control). Immunohistochemical staining was performed using the biotin-avidin technique with primary mouse antibodies against vimentin. (a) $\times 40$ (b) $\times 100$ (c) $\times 100$ (d) $\times 200$

With increased concentration of SGE $\left(7.2 \mathrm{gL} \mathrm{mL}^{-1}\right)$ in cultivated medium the migration of fibroblasts into the $\mathrm{CHM}$ was reduced in compare to lower concentrations of SGE (Fig. 3) but it was comparable with control (Fig. 4).

\section{DISCUSSION}

Wound healing is a process involving various cells, extracellular matrix components as well as many biologically active substances in order to replace and regenerate damaged or lost tissues. This process is often negatively affected by microbial infection or pathological condition which leads into a development of chronic wound. In many cases, standard therapeutic approaches fail to produce expected results. At present, the great hope is given to larval therapy (Sherman, 2009). It was shown that ES products from maggots contain proteinases which are involved in the remodeling of extracellular matrix components. The proteinases cause the lysis of fibrin/ECM, releasing proliferative effectors, e.g., fibronectin fragments, which cause the enhanced healing effects (Horobin et al., 2003). Previous studies demonstrated successful utilization of sterile maggots of greenbottle blowfly $L$. sericata to improve wound healing (Wolff and Hansson, 2003; Pascal et al., 2007; Dumville et al., 2009). In present study, the effect of extract obtained from salivary glands of $L$. sericata on the human dermal fibroblasts proliferation within CHM in vtro was observed.
Our results from immunohistochemical examination revealed the modification of fibroblast migration into the CHM in a dose-dependend manner when compared with intact control after 5 and 10 days of cultivation in vitro. These results are in accordance with the findings obtained by Horobin and co-workers who used $2 \mathrm{D}$ and $3 \mathrm{D}$ in vitro models to evaluate the effect of ES products from maggots on migration of dermal fibroblasts and their interaction to ECM components (Horobin et al., 2005; 2006).

Based on these findings, it may be concluded that ES products promote the re-organization of basic components of CHM by the presence of maggot's proteinases, especially serineproteinase, metalloproteinase and aspartylproteinase that play a role in remodeling of extracellular matrix, both in vitro and in vivo. ES products may also enhance the production of various enzymes by cultured fibroblasts (Chambers et al., 2003; Beasley and Hirst, 2004). Moreover, other bioactive substances which are present in ES products may affect the proliferation of fibroblasts or may lead into direct activation of cell surface receptors and thus increase the cell-ECM interactions (Horobin et al., 2006). For instance, fatty acids (mainly arachidonic acid) and their metabolites are well-known mediators of several cellular events including cell growth (Zhang et al., 2010). Similarly, proteolytic breakdown products of collagen and fibronectin induce fibroblast chemotaxis and migration (Livant et al., 2000).

In summary, our results demonstrated beneficial effect of SGE from larvae of L. sericata on proliferation of human fibroblasts within CHM under in vitro conditions. However, more in vitro and in vivo studies are needed to fully confirm the efficacy of SGE in healing of chronic wounds. In addition, this extract may be used in a field of tissue engineering for preparing of skin substitutes.

\section{ACKNOWLEDGEMENT}

This study was funded by the Operational Program Research and Development and co-financed by the European Fund for Regional Development (EFRD). Grant: ITMS 26240220020 "Establishment of biotherapeutic facility and technology proposal for production and development of bio-drugs".

\section{REFERENCES}

Beasley, W.D. and G. Hirst, 2004. Making a meal of MRSA-the role of biosurgery in hospital-acquired infection. J. Hosp. Infect., 56: 6-9. PMID: 14706264 
Chambers, L., S. Woodrow, A.P. Brown, P.D. Harris and D. Phillips et al., 2003. Degradation of extracellular matrix components by defined proteinases from the greenbottle larva Lucilia sericata used for the clinical debridement of nonhealing wounds. Br. J. Dermatol., 148: 14-23. DOI: 10.1046/j.1365-2133.2003.04935.x

Dumville, J.C., G. Worthy, M.O. Soares, J.M. Bland and N. Cullum et al., 2009. VenUS II: A randomised controlled trial of larval therapy in the management of leg ulcers. Health. Technol. Assess., 13: 1-182. PMID: 19925723

Falch, B.M., L.D. Weerd and A. Sundsfjord, 2009. Maggot therapy in wound management. Tidsskr. Nor. Laegeforen., 129: 1864-1867. PMID: 19844278

Gupta, A., 2008. A review of the use of maggots in wound therapy. Ann. Plast. Surg., 60: 224-227. DOI: 10.1097/SAP.0b013e318053eb5e

Horobin, A.J., K.M. Shakesheff, S. Woodrow, C. Robinson and D.I. Pritchard, 2003. Maggots and wound healing: an investigation of the effects of secretions from Lucilia sericata larvae upon interactions between human dermal fibroblasts and extracellular matrix components. Br. J. Dermatol., 148: $923-933$. DOI: $10.1046 /$ j.13652133.2003.05314.x

Horobin, A.J., K.M. Shakesheff and D.I. Pritchard, 2005. Maggots and wound healing: an investigation of the effects of secretions from Lucilia sericata larvae upon the migration of human dermal fibroblasts over a fibronectin-coated surface. Wound. Repair. Regen., 13: 422-433. DOI: 10.1111/j.1067-1927.2005.130410.x

Horobin, A.J., K.M. Shakesheff and D.I. Pritchrd, 2006. Promotion of Human Dermal Fibroblast Migration, Matrix Remodelling and Modification of Fibroblast Morphology within a Novel 3D Model by Lucilia sericata Larval Secretions. J. Invest. Dermatol., 126: 1410-1418. DOI: 10.1038/sj.jid.5700256

Livant, D.L., R.K. Brabec, K. Kurachi, D.L. Allen and Y. Wu et al., 2000. The PHSRN sequence induces extracellular matrix invasion and accelerates wound healing in obese diabetic mice. J. Clin. Invest., 105: 1537-1545. DOI: 10.1172/JCI8527
Pascal, S., E.J. Cathrien, V.D. Louk, O. Jacques, 2007. Maggot debridement therapy of infected ulcers: Patient and wound factors influencing outcome - a study on 101 patients with 117 wounds. Ann. R. Coll. Surg. Engl., 89: 596-602. DOI: 10.1308/003588407X205404

Plas, V.D.M.J., M. Baldry, J.T.V. Dissel, G.N. Jukema and P.H. Nibbering, 2009a. Maggot secretions suppress pro-inflammatory responses of human monocytes through elevation of cyclic AMP. Diabetologia, 52: 1962-1970. PMID: 18201474

Plas, M.J.A.V.D., J.T.V. Dissel1, P.H. Nibbering, 2009b. Maggot secretions skew monocytemacrophage differentiation away from a proinflammatory to a pro-angiogenic type. PLoS One, 4: e8071. PMID: 19956650

Repiska, V., I. Varga, I. Lehocky, D. Böhmer and M. Blasko et al., 2010. Biological and morphological characterization of human neonatal fibroblast cell culture B-HNF-1. Biologia, 65: 919-924. DOI: 10.2478/s11756-010-0095-6

Sherman, R. A. M. J. R. Hall and S. Thomas, 2000. Medicinal maggots: An ancient remedy for some contemporary afflictions. Ann. Rev. Entomol., 45: 55-81. DOI: 10.1146/annurev.ento.45.1.55

Sherman, RA., 2009. Maggot therapy takes us back to the future of wound care: New and improved maggot therapy for the 21 st century. J. Diabetes. Sci. Technol., 3: 336-344. PMID: 20144365

Vojtassak, J., D. Bakos, L. Danihel, J. Kristin and D. Bohmer et al., 2001. In vitro cytotoxicity testing of coladerm membrane. Cell Tissue Bank., 2: 225233. DOI: $10.1023 / \mathrm{A}: 1021146217176$

Whitaker, I.S., C. Twine, M.J. Whitaker, M. Welck and C.S. Brown et al., 2007. Larval therapy from antiquity to the present day: mechanisms of action, clinical applications and future potential. Postgrad. Med. J., 83: 409-413. DOI: 10.1136/pgmj.2006.055905

Wolff, H. and C. Hansson, 2003. Larval therapy - an effective method of ulcer debridement. Clin. Exp. Dermatol., 28: 134-137. DOI: 10.1046/j.13652230.2003.01226.X

Zhang, Z., S. Wang, Y. Diao, J. Zhang and D. Lv, 2010. Fatty acid extracts from Lucilia sericata larvae promote murine cutaneous wound healing by angiogenic activity. Lipids Health. Dis., 9: 1-9. PMID: 20211009 